\title{
Dissemination Techniques for STEM Intervention Programs
}

\author{
Lawrence O. Flowers, PhD \\ Associate Professor of Biology \\ Biology Department \\ Livingstone College \\ 701 W Monroe St, Salisbury, NC 28144, USA
}

\begin{abstract}
Dissemination is the act of dispersing information or materials to a broader audience to improve awareness and knowledge. Dissemination protocols are equally paramount for science, technology, engineering, and mathematics (STEM) intervention programs. STEM intervention programs are typically multifaceted academic programs that focus on paramount objectives designed to ultimately enhance the nation's economic competitiveness on the global stage. Additionally, a key focus of SIPs is to broaden the participation of underrepresented groups to improve diversity in STEM education and careers. Effective dissemination schemes are advantageous to directors and principal investigators interested in their program's sustainability as well as to achieve the broader impacts of the STEM program. Also, the calculus behind crafting effective dissemination plans is that it contributes to the overall success of the SIP. The cost-effective dissemination framework discussed in this article is designed explicitly for STEM intervention programs but can also apply to a variety of organizations and settings. This article offers practical and scalable dissemination strategies for faculty, administrators, and intervention program support staff. Also included in this article is an example of a logic model.
\end{abstract}

Keywords: dissemination, intervention programs, online, STEM

\section{Introduction}

Dissemination in a scholarly context is generally defined as the process of dispersing information to relevant audiences. Sharing qualitative and quantitative methodologies, mixed methods designs, research findings, policies, best practices, institutional information, demographic statistics, organizational management schemes, product information, and participant outcomes is a hallmark of every organization at every level of government, education, and business. This article focuses specifically on dissemination activities in an academic setting, specifically science, technology, engineering, and mathematics (STEM) intervention programs or SIPs. One of the reasons for poor dissemination outcomes observed with many SIPs is that administrators or principal investigators of these programs lack the specialized marketing and public relations training to successfully engage in a coordinated and systemic dissemination campaign. This article will offer helpful strategies SIP staff can employ to improve the dissemination aspects of their program. An operational definition of SIPs is provided in the next section. The first step to improve dissemination outcomes is to change the mindset of SIP directors and institutional administrators. As a program director you must see yourself as more than just a content expert managing intricate components of an integrated network. You must also think of yourself as a marketing manager. Dissemination involves rigorously transmitting information about the positive aspects of your program to your stakeholders (i.e., customers).The dissemination of experimental data, implementation activities, best practices, scholarly publications is a step in the right direction.

\section{STEM Intervention Programs}

STEM intervention programs (SIPs) are typically implementation programs sponsored by academic institutions such as secondary schools, colleges/universities, and professional schools that are designed to enhance STEMrelated affective, behavioral, or cognitive skills of students or trainees (Rincon\& George-Jackson, 2016a; Rincon\& George-Jackson, 2016b). SIPs are the primary drivers of increasing representation and vocational support of underrepresented groups in STEM education and careers. 
There are many different types of SIPs reported in the literature, and while they share similar program goals, unique SIPs target different demographic groups (Maton et al., 2016; Rakich\& Tran, 2016; Ramsey, Betz, \& Sekaquaptewa, 2013). SIPs have received attention in the last decade as the nation seeks to develop qualified, competent scientists, technologists, and engineers who will lead the country into the next decade through entrepreneurial innovation and fill the enormous number of new jobs that are estimated according to federal agencies (Langdon, McKittrick, Beede, Khan, \&Doms, 2011).Billions in funding have been invested in STEM education initiatives to improve student outcomes mainly in the areas of academic and professional achievement. Minorities, women, and students with disabilities have received significant funding, since these groups are severely underrepresented in the STEM enterprise. While the data demonstrates that positive gains have been made in many paramount areas, much work needs to be done on many fronts (National Center for Science and Engineering Statistics, 2017).

SIPs largely focus on activities that enhance learning, training, teaching, graduation rates, employment outcomes, and efficacy. Broadening participation in STEM is also a major focus area of SIPs in the United States. SIPs are firmly grounded in theory or canonical conceptual frameworks. Implementation activities in SIPs are evidencebased and often reflect the best practices in a particular field or a conflation of multiple disciplines. Successful SIPs also employ program evaluation strategies that typically involve sound research methodologies and statistically valid data collection methods in which vital information is collected from participants and program staff during and after the SIP. Data collection tasks are then followed by rigorous data analytical procedures to elucidate key findings.

Highly structured SIPs have the potential to impact current pedagogical strategies and organizational policy at all levels. The advancement of knowledge and production of basic research findings is also a collateral consequence of an elegant SIP. Novel data can then be used by other researchers to expand the knowledge base and improve society. It is clear from the overview that dissemination activities are an essential component of information exchange for SIPs. Both small-scale and large-scale dissemination actionable measures should target precise communities and stakeholders likely to benefit from the disseminated material. While some researchers disagree about the effects of information dissemination efforts and highlight failed attempts to achieve adoption of evidence-based instructional approaches, most agree that if employed correctly under various temporal parameters that the distribution of educational materials can have positive and lasting effects on its recipients (Gannaway, Hinton, Berry, \& Moore, 2013). Froyd et al. 2017 assert that it is important for program developers to adopt a dissemination approach that is characterized by frequently interaction with targeted populations, and one that seeks feedback from the desired populations in attempt to adjust informational exchange that is aligned with the evolving needs of the beneficiaries.

\section{Dissemination Plan}

A very important first step when articulating the dissemination component for your institution, STEM intervention program, or grant proposal is the production of a thorough dissemination plan. Before you begin constructing your dissemination plan, you should first discuss and record answers to a series of basic questions: Why are dissemination activities important for my program?, What are the characteristics of my dissemination audience?, What resources or funding are available for the dissemination component of the project? What are the most cost-effective dissemination procedures available for my program? What parameters or indicators constitute a successful dissemination campaign? Recommendations to the above issues are found in this article to aid the brainstorming process.

In your dissemination plan, list measurable summative goals and incremental indicators of success. Consider the advantages and disadvantages of each strategic element of your plan and eliminate superfluous and costly approaches. At each phase of the dissemination plan your audience or stakeholders must be carefully targeted. Data shows that certain demographics may benefit from general and specialized forms of program information. The nature of the communication platform (e.g., mobile application, e-mail, standard mail service, etc.) may also be more appropriate for certain groups. Elicit beneficial feedback from administrators, faculty, and others to make sure that your dissemination plan is valid and feasible given the unique characteristics of your organization or program. 
The inclusion of specific indicators of dissemination success at distinct time periods is very important and must be highlighted in the dissemination plan. A detailed timeline of dissemination tasks linked to key personnel will ensure that assignments are being completed on time by the most qualified individual within the organization. SIP administrators should not rule out the possibility of hiring a professional PR (public relations) firm for initial consultations or for completing major information dissemination tasks. Collaborations with PR firms can be quite expensive and therefore may not be feasible for most program budgets. A cheaper alternative to hiring aPR firm is to simply utilize a press release service to disseminate SIP-related information to specific groups or to the general public. Prices range for $\$ 40$ to $\$ 1,000$ depending on the coverage of your information. Press release companies provide a cost-effective way to share information to particular audiences. Following the information distribution process, most press release companies provide their clients with records showing exactly where the information was sent (e.g., news outlets, publications) which will aid in the evaluation of dissemination methods. Table 1 provides recommendations of dissemination strategies that can be employed to complete the dissemination phase of your SIP. In terms of disseminating data or research findings keep in mind that term data is not an all-encompassing term. There are different types of data that are generated during the course of a SIP such as social science data, scientific data, evaluation data, and general program data. Understanding that a SIP may generate several different types of data is important because different types of data can be forwarded to different stakeholders.

Too often data dissemination plans only consider social science data or evaluation data when deciding which data to distribute. Other forms of data are equally or more important for some interested parties. For example, if your SIP focuses on examining the effects of a novel pedagogical technique at the undergraduate level then at the completion of your program you may want to share the technique with the educational community by posting the instructional method on the National Science Digital Library (NSDL) Pathway for biology education. Alternatively, if your program involves the collection of social science data, such as hierarchical linear regression modeling in which you examine engineering students' perceptions to active learning (Finelli et al., 2018), then you may want to consider submitting your data to the Berkeley Initiative for Transparency in the Social Sciences (BITSS). Keep in mind that storage of your data on online repositories may negatively affect your budget. While some repositories are free some require one-time or even recurring monthly fees. Always conduct the necessary research to ensure that the data storage site is aligned with your program's budget. The benefits of storing and sharing your data are numerous. First, storage on a secure server ensures that your data are safe from hackers, but most importantly when your data is stored on an accessible electronic platform you allow others in the educational community access to your results.

This type of transparency is consistent with the tenets of universal information exchange and will allow other researchers the opportunity to validate your results and to build new connections and scholarly insights. It is also suggested that your dissemination plan contain projected numbers of scholarly products. Simply, stating that you plan to publish an article is insufficient. You must document the actual number of publications and specific journals or other media sources you plan to target. Further, you can create a separate scholarly publication table that includes not only the number of publications but also includes a working draft of publication titles and abstracts. The title and abstract may change however the initial structure of the publication table will strengthen the dissemination plan and the overall grant proposal.

\section{Customized Website}

Most dissemination strategies for SIPs involve the production of a customized website for the program. According to a recent report, a customized website is still the preferred platform over emerging social media dissemination outlets (Cooper, 2014). The term customized website can mean several different things. It may mean that you create a webpage that is associated with a larger organization (e.g., college website or department website) or it may mean that you create a stand-alone website. Regardless, of how you structure your website, creating a website or webpage for your SIP is an excellent idea for a number of reasons. Creating your own website provides control over the type of content that is disseminated. For example, some of the data that is generated during your program may not be compelling enough for a scholarly publication but may enhance the quality of your website. Taddeo and Barnes (2016) purported a website development framework that may be instrumental when designing effective education websites. Recommendations in the article provide helpful techniques to consider during the design and maintenance of your customized website. 
Additionally, website development and website hosting while not unduly expensive must be considered as you prepare your budget. Regular maintenance will ensure that the information on your website is current. Rather than upload your data on a commercial online data repository, consider inserting a data upload feature on your own website. Having this feature would also provide greater control over data access. Most online services are free or allow users to pay either monthly or yearly fees to access data, which means that the data is available to anyone. By creating your own site with this data management feature you can place restrictions on who has access to the data and knowledge generated from your SIP. Consult with legal counsel when designing intellectual property rights parameters regarding data access, sharing, and ownership. Adding in website analytical tools to track website usage at the geographical level will alert program directors to trends in online material consumption and inform information outreach responsibilities. Consistent with data management rules (Van den Eynden, Corti, Woollard, \& Bishop, 2009) you must explicitly outline in your dissemination plan how you plan to share data with unique communities.

\section{SIP Videos}

Videos are a unique way to capture and share actual interactions between SIP staff and participants. Videos also demonstrate the precise level of engagement with program resources and implementation activities. Videos can also be designed to display data generated from social science research, explain a novel pedagogical strategy, or provide instructional support regarding a scientific laboratory technique. Outsourcing the video production is a good idea when quality is desired, however, due to the latest video technology found on smartphones creating your own video is becoming a much easier task. Several video sharing websites are included in Table 1. Conversely you could simply disseminate your video to select viewers. The dissemination plan should also include the costs of each activity. The dissemination strategy explicated in Table 1 is designed to distribute novel experimental data derived from research projects and ultimately increase the knowledge base regarding established and emergent disciplines. The multilayered approach will include the standard strategies such as research conferences, websites, scholarly publications as well as other approaches utilizing the technological advancements commonly consumed in society.

It's a great big world out there. Think globally when designing dissemination plans. Science at its core is a collaborative endeavor, the dissemination of social science data, scientific data, evaluation data, and program data is aligned with those most fundamental tenets. Dissemination planning is essential. The success you will enjoy after the project is completed will be attributed to the critical planning strategy you created prior to receiving the award.

\section{Evaluate Outcomes}

Conducting formative and summative assessments to ensure compliance with dissemination procedures and to confirm success is paramount. When performing the evaluation plan you want to generate descriptive statistics as a way to measure success. Remember, during the planning phase you created indicators and benchmarks for particular dissemination components. At this stage, you are simply verifying whether you have met your mark or fallen short, at which point remediation strategies can be executed. Confirmation of dissemination goals could involve the use of emails or telephone calls to confirm that the desired group has received the information. It could also involve website usage statistics, number of views on YouTube, number of retweets, or the number of times your information has been shared with others. Again, if you do not meet expectations during the initial formative assessment, develop additional approaches and perform the assessment again. This remediation cycle is a standard practice in the educational community.

\section{Dissemination Logic Model}

In general, logic models are schematic diagrams that outline key components of paramount organizational processes and have been utilized for a wide variety of educational endeavors (Brown, 2012; Lawton, Brandon, Cicchinelli, \& Kekahio, 2014; Strycker, 2016; Weiss, 1972). The use of logic models has surged in recent years as administrators are learning the value of creating road maps for executing successful operations (Helitzer et al., 2010; Martin \& Carey, 2014).The visual nature of the logic model provides SIP architects and program evaluators a straightforward mechanism for planning and evaluating core elements of the dissemination plan. The logic model should depict the available resources you have or will obtain in the future to carry out your dissemination objectives. 
Additionally, the logic model should illustrate specific tasks, target audiences, monitoring protocols, and desired results. The desired results must be aligned with the overall strategic vision of the SIP and mission of the affiliate institution. It should clearly convey the sequential nature of the dissemination process.

Evaluation efforts may lead to minor or major changes in the dissemination strategies and goals in order to remain compliant to the overall institutional mission. A comprehensive dissemination logic model is best achieved when multiple members of the project team collaborate to complete this task. The inclusion of many viewpoints will ensure a more thorough and feasible dissemination logic model and may enhance the motivation of the entire project team. Avoid nimiety in the logic model and only specify pertinent inputs (e.g., funding), outputs (e.g., participants, virtual methods, publications), and outcomes (e.g., advancement of knowledge). Figure 1 shows the logic model for specific dissemination activities.

In a logic model, inputs clarify the initial investment and contributions that must be expended to catalyze the initiative. For the dissemination phase, this includes both human and technological resources. Some logic models also contain an entry before the inputs parameter called the situation, problem, institutional policy, or organizational priorities. This section is used to provide context and a justification for the inputs necessary to begin and should be aligned with the subsequent outputs, outcomes, and impacts. Outputs define the participants, tasks, and interaction products that characterize the SIP. This area illuminates the proposed program activities, highlights the target audience (e.g., students, investors), and presents a snapshot of the measurable products that resulted from the interaction with the participants, stakeholders, and the program activities. If the outputs meet expectations, typically favorable outcomes are produced. Outcomes are usually divided into three groups: short-term, intermediate, and long-term. Outcomes must be measurable and should be framed within the context of the overall goals of the SIP. Impacts are distant potential consequences that are more difficult to measure and reflect societal goals on a national or international scale. Since disparate SIPs have common objectives, the outcome and impacts sections for different SIPs may be similar.

Logic models express the casual relationships and interdependence of SIP program components. These types of illustrations are an excellent way to visualize what needs to get done in order to achieve successful outcomes and to assess efficacy at each stage of the program. Execution and performance indicators can be extracted so that analytical measurements can be performed and evaluated. Logic models are not perfect schematics and have several limitations.

SIPs are intricately complex endeavors that can't possibly be captured on any visual linear schematic. Moreover, as many program directors and principal investigators already understand, successful SIPs are often influenced by external factors that were nearly impossible to predict at the outset of the program. Also, the sequential nature and limited space requirements due to page limitations in federal proposals make it impossible for any logic model to represent the true nature of the actual program. Continuous formative evaluations of the dissemination plan will allow for adjustments to be made to counteract any unforeseen or unpredictable forces that may impact your SIP's dissemination strategy.

The pedagogical, political, educational, and societal implications of disseminated information may be far reaching. SIP evidence-based knowledge may lead to the development of innovative teaching strategies or could lead to the adoption of sweeping institutional policy that may result in gains in broadening participation for underrepresented groups. The submission of annual reports is a standard feature of nearly every federal grant program and is therefore a major dissemination product. Closely adhere to the guidelines and submission specifications of the funding agency when completing required annual reports. Contact agency officials if you are unclear about the correct submission portal to ensure that you submit the requisite documentation on time. There are a number of online resources that are readily accessible as you embark on your journey to complete a dissemination plan. One helpful resource proffered by Harmsworth \& Turpin (2000) will serve as an excellent starting point. 


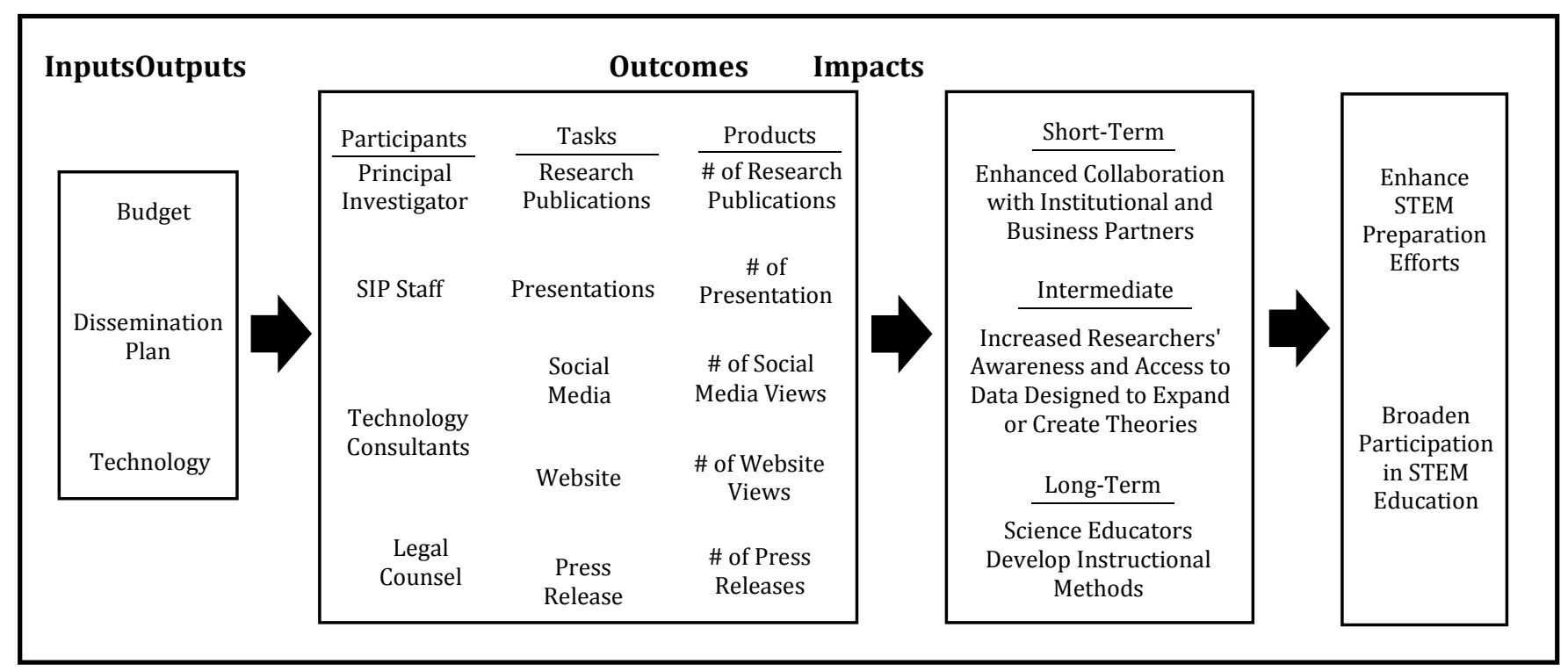

Figure 1-Dissemination Phase Logic Model

\section{Dissemination Strategies}

Table 1 shows a few helpful tips that may be employed to adhere to information transmission objectives. The list is by no meansall-inclusive; yetit is adequately comprehensive to enlighten your SIP dissemination plan. Depending on the items on the list, some methods will be better suited for particular programs. Strategies in Table 1 have been systematically arranged into four categories (e.g., publications, presentations, media, other) based on activity for easier manipulation. This is not a rigid classification system. Some items in Table 1 can fit in multiple categories depending on the context.

Online or virtual dissemination methods are the preferred methods of information distribution in the $21^{\text {st }}$ century and offer several advantages to principal investigators. Previous data asseverates that virtual transmission methods are more beneficial than traditional communication approaches. Regardless of the dissemination strategy you employ always remember to acknowledge the funding agency or principle benefactor responsible for providing financial support or other type of indispensable provisions for your SIP. An often overlooked tactic in terms of dissemination is the compiling and dispersal of SIP-related photos. Again, smartphones and mobile applications have made capturing and sorting photos painstakingly easy. Photos are becoming a valuable component of accreditation portfolios and evidence of institutional action. Many federal funding agencies now have unique uploading features on their website for photos.

Establishing partnerships with similar businesses and SIPs around the country is also an excellent strategy to improve your dissemination infrastructure. It will also allow you to disseminate data and other information to faculty and businesses around the country that have similar interests. In some cases, before you disseminate your work to the public you may want to either copyright, trademark, or patent your work so that you can retain intellectual property rights and deter others from taking credit for your hard work.

\section{Conclusions}

Essentially, a SIP consists of multiple components including the actual implementation activities, data management procedures, and the evaluation of program efficacy. The point is that each component of the SIP has intellectual value that can be disseminated. When you think of the dissemination phase as potentially assisting others, you realize that any type of knowledge you generate regarding best practices can and should be shared to help specific stakeholders, beneficiaries, and other groups. This article presents a collection of lucubrations of successful dissemination strategies for SIPs. In academic environments, SIP viability and sustainability is dependent on continuous funding. Perpetual funding is dependent on the ability to demonstrate the program's success and impact on the participants and institutional benefactor. 


\section{Acknowledgements}

This work was supported by grants funded by the National Science Foundation (HRD-1533536 and HRD1505098). All of the recommendations and other information contained in this article are those of the author and do not necessarily reflect the National Science Foundation.

\section{Table 1 - STEM Intervention Program Dissemination Strategies}

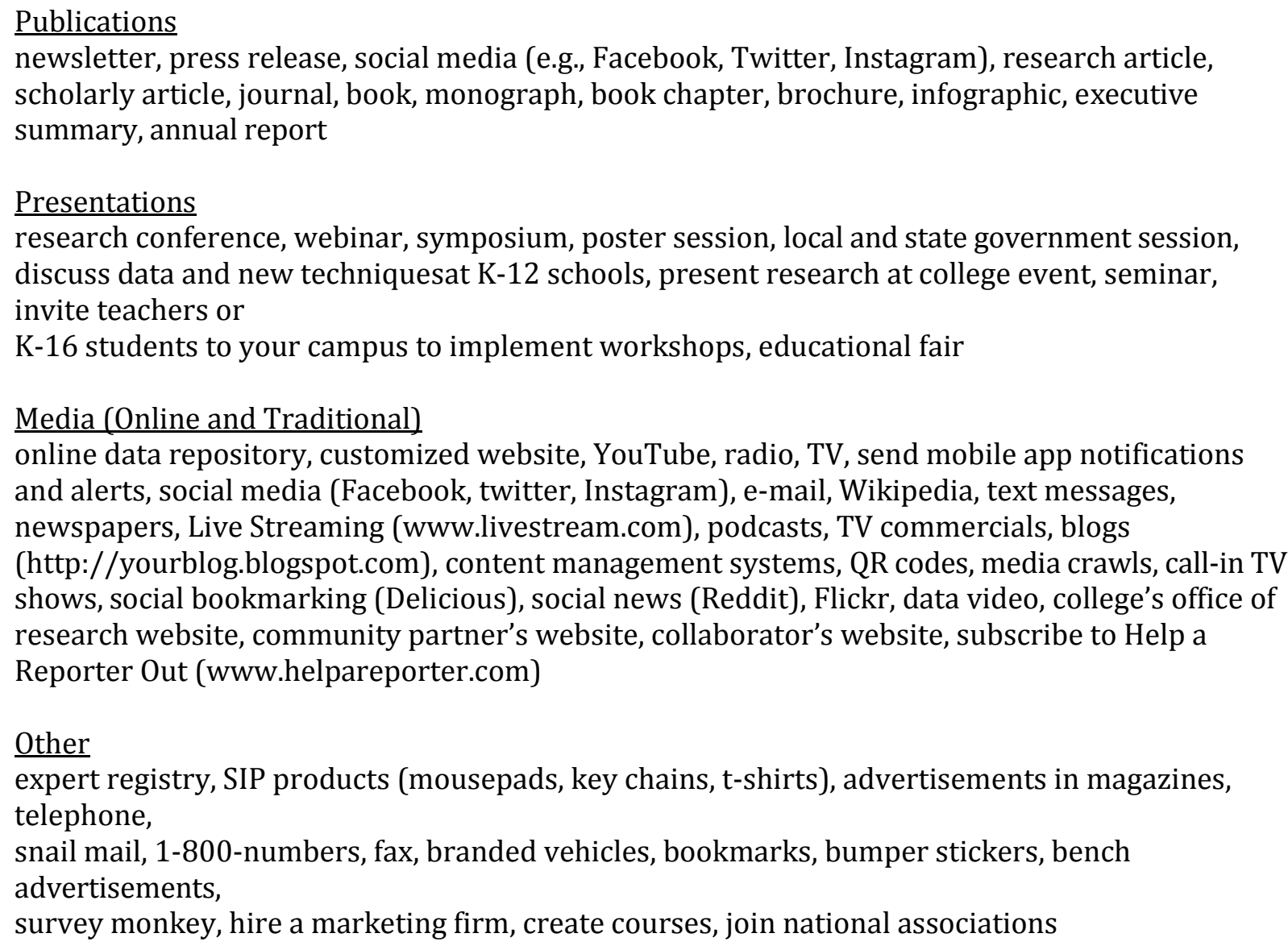

\section{References}

Brown, C. (2012). Use of logic models to plan and assess graduate internship experiences.TechTrends: Linking Research and Practice to Improve Learning, 56, 37-43.

Cooper, A. (2014). The use of online strategies and social media for research dissemination in education.Education Policy Analysis Archives, 22(88). Available from: http://dx.doi.org/10.14507/epaa.v22n88.2014.

Finelli, C., Nguyen, K., DeMonbrun, M., Borrego, M., Prince, M., Husman, J., Henderson, C., Shekhar, P., \& Waters, C. (2018).Reducing student resistance to active learning: Strategies for instructors.Journal of College Science Teaching, 47, 80-91.

Froyd, J., Henderson, C., Cole, R., Friedrichsen, D., Khatri, R., \&Stanford, C. (2017). From dissemination to propagation: A new paradigm for education developers. Change: The Magazine of Higher Learning, 49, $35-42$.

Gannaway, D., Hinton, T., Berry, B., \& Moore, K. (2013). Cultivating change: Disseminating innovation in higher education teaching and learning.Innovations in Education and Teaching International, 50, 410-421.

Harmsworth, S., \& Turpin, S. (2000).Creating an effective dissemination strategy an expanded interactive workbook for educational development projects.TQEF National Co-ordination Team. Available from: http://www.innovations.ac.uk/btg/resources/publications/dissemination.pdf. 
Helitzer, D., Hollis, C., de Hernandez, B., Sanders, M., Roybal, S., \& Van Deusen, I. (2010). Evaluation for community-based programs: The integration of logic models and factor analysis. Evaluation and Program Planning, 33, 223-233.

Langdon, D., McKittrick, G., Beede, D., Khan, B., \&Doms, M. (2011).STEM: Good jobs now and for the future (ESA Issue Brief \#03-11). Washington, DC: U.S. Department of Commerce.

Lawton, B., Brandon, P.R., Cicchinelli, L., \&Kekahio, W. (2014).Logic models: A tool for designing and monitoring program evaluations. (REL 2014-007). Washington, DC: U.S. Department of Education, Institute of Education Sciences, National Center for Education Evaluation and Regional Assistance, Regional Educational Laboratory Pacific. Available from:http://ies.ed.gov/ncee/edlabs.

Martin, I., \& Carey, J. (2014).Development of a logic model to guide evaluations of the ASCA national model for school counseling programs.Professional Counselor, 4, 455-466.

Maton, K., Beason, T., Godsay, S., Domingo, M., Bailey, T., Sun, S., \&Hrabowski, F. (2016). Outcomes and processes in the Meyerhoff scholars program: STEM PhD completion, sense of community, perceived program benefit, science identity, and research self-efficacy. CBE - Life Sciences Education, 15, 48.

National Center for Science and Engineering Statistics. (2017). Women, minorities, and persons with disabilities in science and engineering: 2017. Special Report NSF 17-310. Arlington, VA.

Rakich, S., \& Tran, V. (2016). A balanced approach to building STEM college and career readiness in high school: Combining STEM intervention and enrichment programs. European Journal of STEM Education, 1, 59.

Ramsey, R., Betz, D., \&Sekaquaptewa, D. (2013).The effects of an academic environment intervention on science identification among women in STEM.Social Psychology of Education: An International Journal, 16, 377-397.

Rincon, B., \& George-Jackson, C. (2016a).Examining department climate for women in engineering: The role of STEM interventions.Journal of College Student Development, 57, 742-747.

Rincon, B., \&George-Jackson, C. (2016b). STEM intervention programs: Funding practices and challenges. Studies in Higher Education, 41, 429-444.

Strycker, J. (2016). Logic models as a way to support online students and their projects. Journal of Educators Online, 13, 135-150.

Taddeo, C., \& Barnes, A. (2016). The school website: Facilitating communication engagement and learning. British Journal of Educational Technology, 47, 421-436.

Van den Eynden, V., Corti, L., Woollard, M., \& Bishop, L. (2009). Managing and sharing data: A best practice guide for researchers. Available from: http://www.data-archive.ac.uk/media/2894/managingsharing.pdf.

Weiss, C. (1972). Evaluation research.Methods for assessing program effectiveness. Englewood Cliffs, NJ:Prentice-Hall. 